\title{
Singular Soliton, Shock-wave, Breather-stripe Soliton, Hybrid Solutions and Numerical Simulations for a (2+1)-Dimensional Caudrey-Dodd-Gibbon- Kotera-Sawada System in Fluid Mechanics
}

Shaohua Liu ( $\nabla$ lsh_bupt@163.com )

Beijing University of Posts and Telecommunications School of Science https://orcid.org/0000-00016042-6941

Bo Tian

Beijing University of Posts and Telecommunications School of Science

\section{Research Article}

Keywords: Fluid mechanics, (2+1)-dimensional Caudrey-Dodd-Gibbon-Kotera-Sawada system, Soliton solutions, Shock-wave solutions, Breather-stripe soliton solutions, Hybrid solutions, Numerical simulations

Posted Date: January 25th, 2022

DOl: https://doi.org/10.21203/rs.3.rs-233477/v1

License: (c) (i) This work is licensed under a Creative Commons Attribution 4.0 International License. Read Full License

Version of Record: A version of this preprint was published at Nonlinear Dynamics on March 28th, 2022. See the published version at https://doi.org/10.1007/s11071-022-07279-9. 


\title{
Singular soliton, shock-wave, breather-stripe soliton, hybrid solutions and numerical simulations for a
} $(2+1)$-dimensional

\section{Caudrey-Dodd-Gibbon-Kotera-Sawada system in fluid mechanics}

\author{
Shao-Hua Liu, Bo Tian* \\ State Key Laboratory of Information Photonics and \\ Optical Communications, and School of Science, Beijing University of Posts \\ and Telecommunications, Beijing 100876, China
}

\begin{abstract}
In this paper, a (2+1)-dimensional Caudrey-Dodd-Gibbon-Kotera-Sawada system is investigated in fluid mechanics via the symbolic computation. With the help of the Hirota method, we derive some singular soliton, shock-wave, breather-stripe soliton and hybrid solutions. Based on the finite difference method, we get some numerical one-soliton solutions. We graphically show the singular and shock-wave solutions, and observe that the singular one-soliton solutions are explosive and unstable, but the shock-wave solutions are nonsingular and stable. We observe that the breatherstripe soliton moves along the negative direction of the $y$ axis, where $y$ is a variable, and the amplitude and shape of the breather-stripe soliton remain invariant during the propagation. We graphically demonstrate the interaction among a rogue wave, a periodic wave and a pair of the stripe solitons: the rogue wave arises from the one stripe soliton; the rogue wave interacts with the periodic wave, the rogue wave splits into two waves and then the two waves merge into a wave; the rogue wave fuses with the other stripe soliton. We graphically present the numerical one-soliton solutions which agree with the analytic one-soliton solutions.
\end{abstract}

*Corresponding author, with e-mail address as tian_bupt@163.com 
Keywords: Fluid mechanics; (2+1)-dimensional Caudrey-Dodd-Gibbon-Kotera-Sawada system; Soliton solutions; Shock-wave solutions, Breather-stripe soliton solutions; Hybrid solutions; Numerical simulations 


\section{Introduction}

Fluid mechanics has been applied in a variety of disciplines such as meteorology, geophysics, biomedical engineering, oceanography and astrophysics [1-4]. In order to gain insight into certain fluid mechanical problems, researchers have focused their attention on some solutions for some nonlinear evolution equations, such as the soliton, breather-wave, periodic-wave and rogue-wave solutions [5-29].

Refs. [30-42] have considered the following (2+1)-dimensional Caudrey-Dodd-GibbonKotera-Sawada system in fluid mechanics:

$$
\left\{\begin{array}{l}
36 u_{t}+u_{x x x x x}+15\left(u u_{x x}\right)_{x}+45 u^{2} u_{x}-5 u_{x x y}-15 u u_{y}-15 u_{x} v-5 v_{y}=0, \\
u_{y}=v_{x}
\end{array}\right.
$$

where $u$ and $v$ are both the differentiable functions with respect to the variables $x, y$ and $t$, and the subscripts indicate the partial derivatives. Darboux transformations and $N$-soliton solutions for System (1) have been derived via the Darboux matrix method, where $N$ is a positive integer [30]. Soliton, rational, triangular periodic, Jacobi and Weierstrass doubly periodic solutions for System (1) have been obtained via the algebraic method [31]. Various soliton, breather and periodic solutions for System (1) have been derived via the long wave limit method [32]. Via the Hirota-Riemann function method [33], quasi-periodic solutions for System (1) have been derived. Symmetry reductions and group-invariant solutions for System (1) have been obtained via the Lou's direct method [34]. Lump, mixed rogue wavestripe soliton, mixed lump-stripe soliton for System (1) have been obtained via the Hirota bilinear method [35]. The higher-order breather, lump and hybrid solutions for System (1) have been obtained via the long wave limit method [36]. The periodic soliton solutions for System (1) have been derived via the Hirota bilinear method [37]. Ref. [38] has derived nontraveling wave solutions for System (1) via the Lie group analysis and exp-function method. Hybrid solutions among lumps, breathers and solitons for System (1) have been obtained via the long wave limit method [39]. Lump solutions have been obtained via the direct method [40]. Symmetry group theorem, some analytic solutions and infinite conservation laws for System (1) have been derived via the improved CK's method [41]. Hybrid solutions comprising the lumps and solitons have been obtained [42].

Under the transformations,

$$
u=2(\ln f)_{x x}, \quad v=2(\ln f)_{x y},
$$

System (1) has been converted into the bilinear form as [39]

$$
\left(5 D_{x}^{3} D_{y}+5 D_{y}^{2}-D_{x}^{6}-36 D_{x} D_{t}\right) f \cdot f=0,
$$

where $f$ is a differentiable function about $x, y$ and $t$, and $D$ is the Hirota bilinear differentiable 
operator, which is defined as [43]

$$
D_{x}^{\iota_{1}} D_{y}^{\iota_{2}} D_{t}^{\iota_{3}}(F \cdot G)=\left.\left(\frac{\partial}{\partial x}-\frac{\partial}{\partial x^{\prime}}\right)^{\iota_{1}}\left(\frac{\partial}{\partial y}-\frac{\partial}{\partial y^{\prime}}\right)^{\iota_{2}}\left(\frac{\partial}{\partial t}-\frac{\partial}{\partial t^{\prime}}\right)^{\iota_{3}} F(x, y, t) G\left(x^{\prime}, y^{\prime}, t^{\prime}\right)\right|_{x^{\prime}=x, y^{\prime}=y, t^{\prime}=t}
$$

with $F(x, y, t)$ being a differentiable function with respect to $x, y$ and $t, G\left(x^{\prime}, y^{\prime}, t^{\prime}\right)$ being a differentiable function about the formal variables $x^{\prime}, y^{\prime}$ and $t^{\prime}$, and $\iota_{1}, \iota_{2}$ and $\iota_{3}$ being three non-negative integers.

However, to our knowledge, singular $N$-soliton solutions, shock-wave solutions, breatherstripe soliton solutions, hybrid solutions among a rogue wave, a periodic wave and a pair of the stripe solitons, and numerical solutions for System (1) have not been investigated. In Section 2, we will construct some singular $N$-soliton and shock-wave solutions for System (1). In Section 3, breather-stripe soliton solutions for System (1) will be established. In Section 4, we will discuss the hybrid solutions among a rogue wave, a periodic wave and a pair of the solitons for System (1). In Section 5, we will obtain certain numerical one-soliton solutions for System (1). In Section 6, conclusions will be drawn.

\section{Singular soliton and shock-wave solutions for System (1)}

\subsection{Singular soliton solutions for System (1)}

In this part, we will construct some singular $N$-soliton solutions for System (1) via the Hirota bilinear method [43]. We use the perturbation method to expand $f$ with respect to a formal parameter $\epsilon$ as

$$
f=1+\epsilon f_{1}+\epsilon^{2} f_{2}+\epsilon^{3} f_{3}+\cdots+\epsilon^{N} f_{N}
$$

where $f_{i}$ 's $(i=1,2, \cdots, N)$ are the real functions of $x, y$ and $t$. Substituting Expression (4) into Bilinear Form (3) and equating the coefficients of the same power of $\epsilon$ to zero, we obtain some soliton solutions of System (1). Thus, when $\epsilon=-1$, singular $N$-soliton solutions can be written as

$$
\begin{aligned}
& u=2(\ln f)_{x x}, \quad v=2(\ln f)_{x y}, \\
& f=1-\sum_{\imath=1}^{N} e^{\eta_{\imath}}+\sum_{\imath<\jmath}^{N} M_{\imath \jmath} e^{\eta_{\imath}+\eta_{\jmath}}-\sum_{\imath<\jmath<\ell}^{N} M_{\imath \jmath} M_{\imath \ell} M_{\jmath \ell} e^{\eta_{\imath}+\eta_{\jmath}+\eta_{\ell}}+\cdots+(-1)^{N}\left(\prod_{\imath<\jmath} M_{\imath \jmath}\right) e^{\sum_{\imath=1}^{N} \eta_{\imath}},
\end{aligned}
$$

where

$$
\begin{aligned}
& \eta_{\imath}=\kappa_{\imath} x+\rho_{\imath} y+\omega_{\imath} t+\eta_{\imath 0}, \quad \omega_{\imath}=\frac{5 \kappa_{\imath}^{3} \rho_{\imath}-\kappa_{\imath}^{6}+5 \rho_{\imath}^{2}}{36 \kappa_{\imath}} \\
& M_{\imath \jmath}=-\frac{\left(\kappa_{\imath}-\kappa_{\jmath}\right)^{6}-5\left(\kappa_{\imath}-\kappa_{\jmath}\right)^{3}\left(\rho_{\imath}-\rho_{\jmath}\right)-5\left(\rho_{\imath}-\rho_{\jmath}\right)^{2}+36\left(\kappa_{\imath}-\kappa_{\jmath}\right)\left(\omega_{\imath}-\omega_{\jmath}\right)}{\left(\kappa_{\imath}+\kappa_{\jmath}\right)^{6}-5\left(\kappa_{\imath}+\kappa_{\jmath}\right)^{3}\left(\rho_{\imath}+\rho_{\jmath}\right)-5\left(\rho_{\imath}+\rho_{\jmath}\right)^{2}+36\left(\kappa_{\imath}+\kappa_{\jmath}\right)\left(\omega_{\imath}+\omega_{\jmath}\right)}
\end{aligned}
$$


$\kappa_{\imath}, \rho_{\imath}, \omega_{\imath}$ and $\eta_{\imath 0}$ are the real constants and $\imath<\jmath<\ell(\ell=3,4, \cdots, N)$.

Assuming $N=1$ in Solutions (5), singular one-soliton solutions can be derived as

$$
\begin{aligned}
& u=2(\ln f)_{x x}=2\left[\ln \left(1-e^{\eta_{1}}\right)\right]_{x x}=-\frac{1}{2} \kappa_{1}^{2} \operatorname{csch}^{2}\left(\frac{\eta_{1}}{2}\right), \\
& v=2(\ln f)_{x y}=2\left[\ln \left(1-e^{\eta_{1}}\right)\right]_{x y}=-\frac{1}{2} \kappa_{1} \rho_{1} \operatorname{csch}^{2}\left(\frac{\eta_{1}}{2}\right),
\end{aligned}
$$

with $\eta_{1}=\kappa_{1} x+\rho_{1} y+\frac{5 \kappa_{1}^{3} \rho_{1}-\kappa_{1}^{6}+5 \rho_{1}^{2}}{36 \kappa_{1}} t+\eta_{10}$.

The physical structure of Solutions (6) is shown in Figs. 1, which shows that Solutions (6)

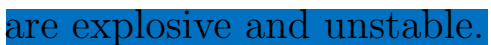

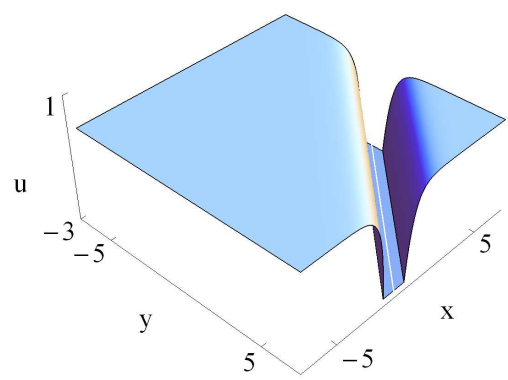

$\left(a_{1}\right) t=-25$

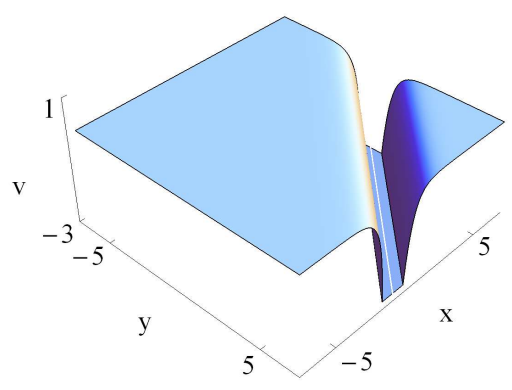

$\left(b_{1}\right) t=-25$

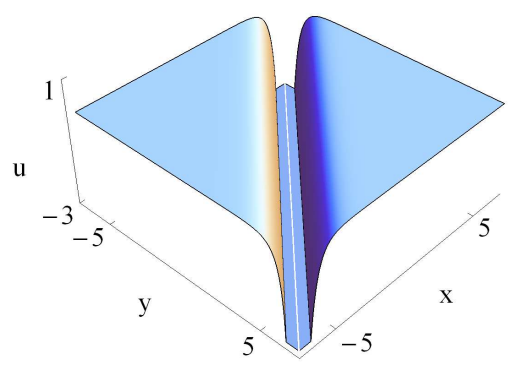

$\left(a_{2}\right) t=0$

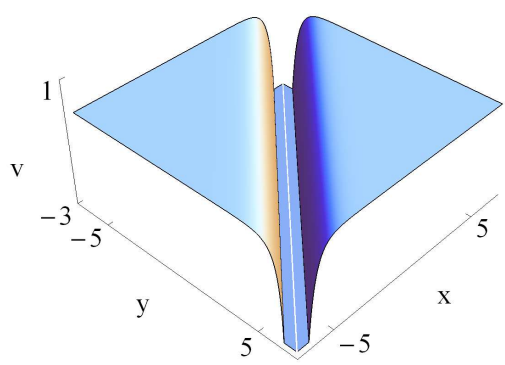

$\left(b_{2}\right) t=0$

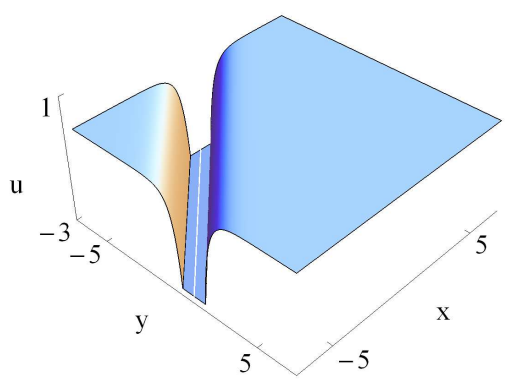

$\left(a_{3}\right) t=25$

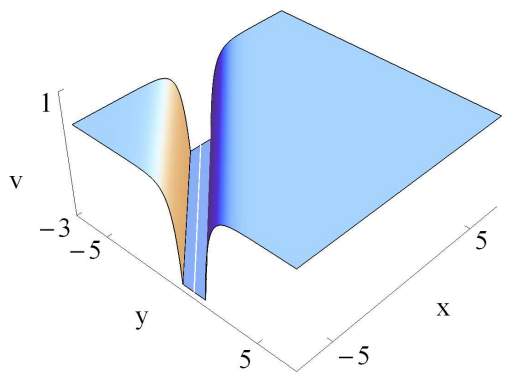

$\left(b_{3}\right) t=25$

Figs. 1. Singular one soliton via Solutions (6) with $\kappa_{1}=\rho_{1}=2$ and $\eta_{10}=0$.

\subsection{Shock-wave solutions for System (1)}

According to Ref. [44], to calculate some shock-wave solutions, we suppose that

$$
u=M \tanh ^{\lambda} \zeta
$$

where $\zeta=a x+b y+c t, M$ is the wave amplitude, $a, b, c$ and $\lambda$ are all the real constants. 
Substituting Expression (7) into $u_{x x x x x}$ and $u^{2} u_{x}$, we derive that

$$
\begin{aligned}
u_{x x x x x}= & a^{5} \lambda M\left[\left(\lambda^{4}-10 \lambda^{3}+35 \lambda^{2}-50 \lambda+24\right) \tanh ^{\lambda-5} \zeta-5\left(\lambda^{4}-6 \lambda^{3}+15 \lambda^{2}-18 \lambda+8\right) \tanh ^{\lambda-3} \zeta\right. \\
& +2\left(5 \lambda^{4}-10 \lambda^{3}+25 \lambda^{2}-20 \lambda+8\right) \tanh ^{\lambda-1} \zeta-2\left(5 \lambda^{4}+10 \lambda^{3}+25 \lambda^{2}+20 \lambda+8\right) \tanh ^{\lambda+1} \zeta \\
& \left.+5\left(\lambda^{4}+6 \lambda^{3}+15 \lambda^{2}+18 \lambda+8\right) \tanh ^{\lambda+3} \zeta-\left(\lambda^{4}+10 \lambda^{3}+35 \lambda^{2}+50 \lambda+24\right) \tanh ^{\lambda+5} \zeta\right], \\
u^{2} u_{x}= & a \lambda M^{3}\left(\tanh ^{3 \lambda-1} \zeta-\tanh ^{3 \lambda+1} \zeta\right) .
\end{aligned}
$$

From Expressions (8), making $\lambda+5=3 \lambda+1$, we get $\lambda=2$. Then, substituting $u=M \tanh ^{2} \zeta$ and $v=-\frac{b M \operatorname{sech}^{2} \zeta}{a}$ into System (1), we obtain that

$$
\begin{aligned}
& 45 a\left(8 a^{4}+6 a^{2} M+M^{2}\right) \tanh ^{7} \zeta-15\left(56 a^{5}+34 a^{3} M+4 a^{2} b+3 a M^{2}+2 b M\right) \tanh ^{5} \zeta-\left[\frac{5 b^{2}}{a}-616 a^{5}\right. \\
& \left.-45 M\left(6 a^{3}+b\right)-100 a^{2} b-36 c\right] \tanh ^{3} \zeta-\left(136 a^{5}+30 a^{3} M+40 a^{2} b-\frac{5 b^{2}}{a}+15 b M+36 c\right) \tanh \zeta=0,
\end{aligned}
$$

which gives us $M=-2 a^{2}$ and $c=\frac{5 b^{2}-76 a^{6}-10 a^{3} b}{36 a}$. Therefore, we obtain the shock-wave solutions for System (1) as

$$
u=-2 a^{2} \tanh ^{2}\left(a x+b y+\frac{5 b^{2}-76 a^{6}-10 a^{3} b}{36 a} t\right), \quad v=2 a b \operatorname{sech}^{2}\left(a x+b y+\frac{5 b^{2}-76 a^{6}-10 a^{3} b}{36 a} t\right) .
$$

Figs. 2 show the propagation of the shock wave. We observe that the amplitude and the shape of the shock wave keep unchanged during the propagation. Therefore, Solutions (9) are the nonsingular and stable solutions.

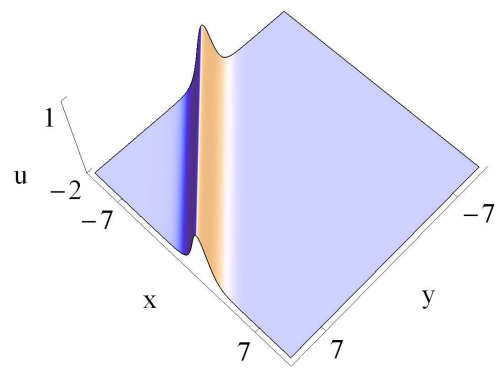

$\left(a_{1}\right) t=-5$

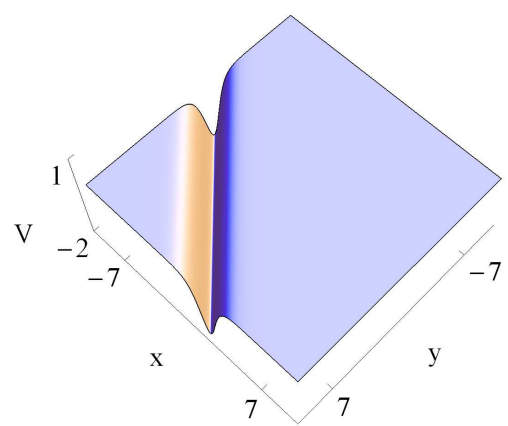

$\left(b_{1}\right) t=-5$

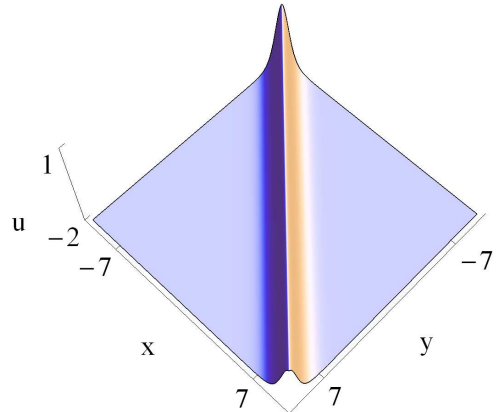

$\left(a_{2}\right) t=0$

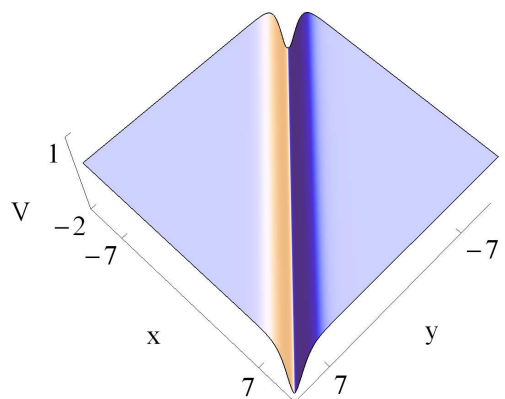

$\left(b_{2}\right) t=0$

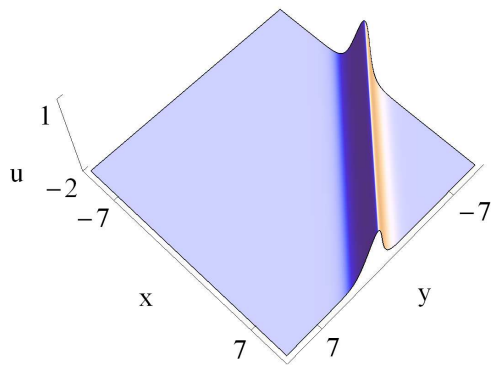

$\left(a_{3}\right) t=5$

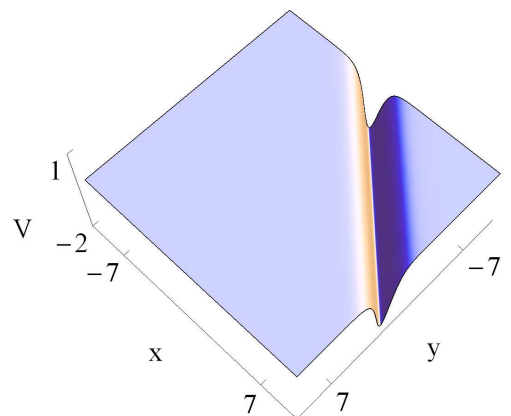

$\left(b_{3}\right) t=5$ 
Figs. 2. Shock wave via Solutions (9) with $a=-b=-1$.

\section{Breather-stripe soliton solutions for System (1)}

To construct the breather-stripe soliton solutions for System (1), inspired by Ref. [45], we assume that

$$
f=s_{1} \cosh \xi_{1}+s_{2} \cos \xi_{2}+s_{3} e^{\xi_{3}}+m_{1}
$$

where $\xi_{\varrho}=k_{\varrho} x+r_{\varrho} y+p_{\varrho} t+q_{\varrho}(\varrho=1,2,3)$, and $k_{\varrho}$ 's, $r_{\varrho}$ 's, $p_{\varrho}$ 's, $q_{\varrho}$ 's, $s_{\varrho}$ 's and $m_{1}$ are the real constants. Substituting Expression (10) into Bilinear Form (3), we derive that

$$
p_{1}=\frac{k_{1}^{5}}{4}, \quad p_{2}=\frac{k_{2}^{5}}{4}, \quad p_{3}=\frac{k_{3}^{5}}{4}, \quad r_{1}=k_{1}^{3}, \quad r_{2}=-k_{2}^{3}, \quad r_{3}=k_{3}^{3} .
$$

According to Expressions (10), (11) and Transformations (2), we get the breather-stripe soliton solutions for System (1) as

$$
\begin{aligned}
u= & \frac{2\left(s_{1} k_{1}^{2} \cosh \xi_{1}-s_{2} k_{2}^{2} \cos \xi_{2}+s_{3} k_{3}^{2} e^{\xi_{3}}\right)}{s_{1} \cosh \xi_{1}+s_{2} \cos \xi_{2}+s_{3} e^{\xi_{3}}+m_{1}}-\frac{2\left(s_{1} k_{1} \sinh \xi_{1}-s_{2} k_{2} \sin \xi_{2}+s_{3} k_{3} e^{\xi_{3}}\right)^{2}}{\left(s_{1} \cosh \xi_{1}+s_{2} \cos \xi_{2}+s_{3} e^{\xi_{3}}+m_{1}\right)^{2}} \\
v= & \frac{2\left(s_{1} k_{1} r_{1} \cosh \xi_{1}-s_{2} k_{2} r_{2} \cos \xi_{2}+s_{3} k_{3} r_{3} e^{\xi_{3}}\right)}{s_{1} \cosh \xi_{1}+s_{2} \cos \xi_{2}+s_{3} e^{\xi_{3}}+m_{1}} \\
& -\frac{2\left(s_{1} k_{1} \sinh \xi_{1}-s_{2} k_{2} \sin \xi_{2}+s_{3} k_{3} e^{\xi_{3}}\right)\left(s_{1} r_{1} \sinh \xi_{1}-s_{2} r_{2} \sin \xi_{2}+s_{3} r_{3} e^{\xi_{3}}\right)}{\left(s_{1} \cosh \xi_{1}+s_{2} \cos \xi_{2}+s_{3} e^{\xi_{3}}+m_{1}\right)^{2}}
\end{aligned}
$$

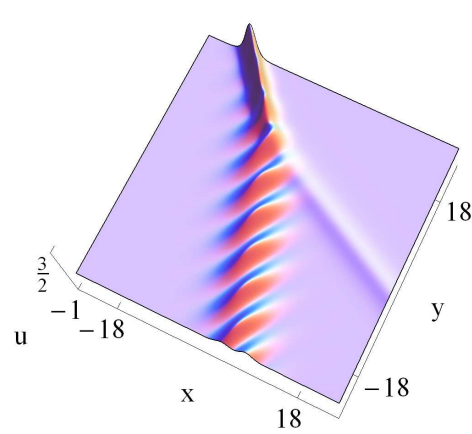

$\left(a_{1}\right) t=-35$

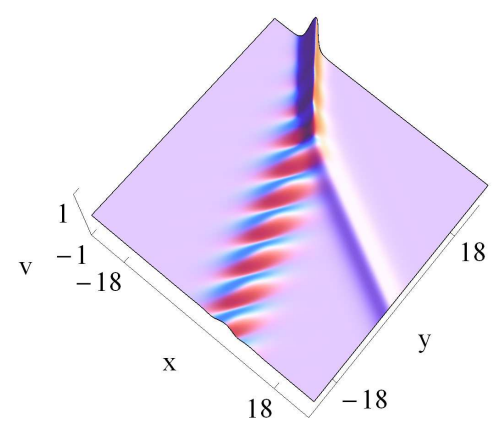

$\left(b_{1}\right) t=-35$

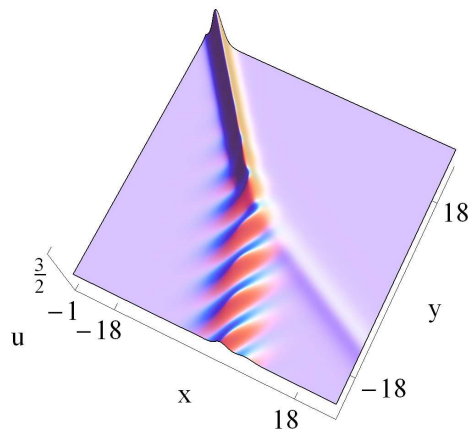

$\left(a_{2}\right) t=0$

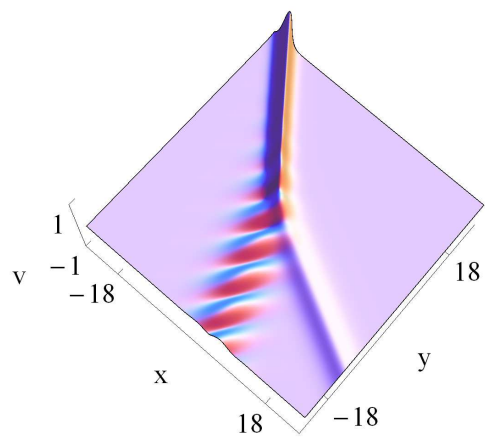

$\left(b_{2}\right) t=0$

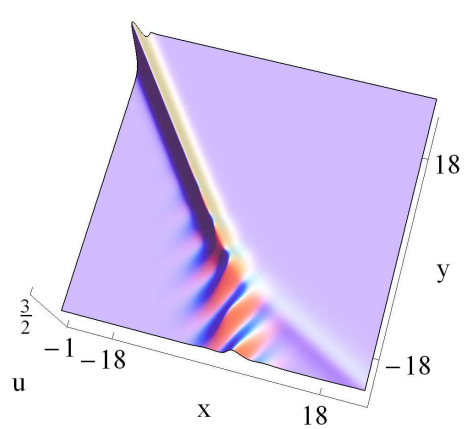

(a $\left.a_{3}\right) t=35$

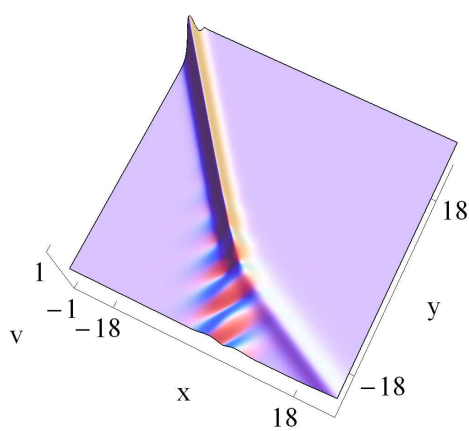

$\left(b_{3}\right) t=35$ 
Figs. 3.

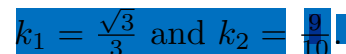

Figs. 3 display the propagation of the breather-stripe soliton. We observe that the breather-stripe soliton moves along the negative direction of the $y$ axis. Amplitude and shape of the breather-stripe soliton remain invariant during the propagation.

\section{Hybrid solutions among a rogue wave, a periodic wave and a pair of the stripe solitons for System (1)}

In order to research the hybrid solutions among a rogue wave, a periodic wave and a pair of the stripe solitons for System (1), motivated by Ref. [46], we assume that

$$
f=s_{4} \xi_{4}^{2}+s_{5} \xi_{5}^{2}+s_{6} \cosh \xi_{6}+s_{7} \cos \xi_{7}+m_{2},
$$

where $\xi_{\sigma}=k_{\sigma} x+r_{\sigma} y+p_{\sigma} t+q_{\sigma}(\sigma=4, \cdots, 7)$, and $k_{\sigma}$ 's, $r_{\sigma}$ 's, $p_{\sigma}$ 's, $q_{\sigma}$ 's, $s_{\sigma}$ 's and $m_{2}$ are the real constants. Substituting Expression (13) into Bilinear Form (3), we obtain that

$$
\begin{aligned}
& k_{4}=\frac{r_{4}}{3 k_{5} k_{7}^{2}} \sqrt{\frac{2 k_{7}^{2}\left(s_{6}^{2}-s_{7}^{2}\right)-4 k_{5}^{2} s_{5} m_{2}}{s_{5} m_{2}}}, \quad p_{4}=-\frac{5 k_{7}^{2} r_{4}}{24 k_{5}} \sqrt{\frac{k_{7}^{2}\left(s_{6}^{2}-s_{7}^{2}\right)-2 k_{5}^{2} s_{5} m_{2}}{2 s_{5} m_{2}}}, \\
& r_{5}=-\frac{3 k_{7}^{2}}{2} \sqrt{\frac{k_{7}^{2}\left(s_{6}^{2}-s_{7}^{2}\right)-2 k_{5}^{2} s_{5} m_{2}}{2 s_{5} m_{2}}}, \quad p_{5}=-\frac{5 k_{5} k_{7}^{4}}{16}, \quad p_{6}=-p_{7}=\frac{k_{7}^{5}}{16}, \quad s_{4}=\frac{9 k_{5}^{2} k_{7}^{4} s_{5}}{4 r_{4}^{2}}, \\
& r_{6}=r_{7}=\frac{k_{7}^{3}}{2}, \quad k_{6}=-k_{7}, \quad k_{5} k_{7} r_{4} \neq 0, \quad s_{5} m_{2}\left[k_{7}^{2}\left(s_{6}^{2}-s_{7}^{2}\right)-2 k_{5}^{2} s_{5} m_{2}\right]>0 .
\end{aligned}
$$

Based on Expressions (13), (14) and Transformations (2), we obtain the hybrid solutions among a rogue wave, a periodic wave and a pair of the stripe solitons for System (1) as

$$
\begin{aligned}
u= & \frac{2\left(2 k_{4}^{2} s_{4}+2 k_{5}^{2} s_{5}+k_{6}^{2} s_{6} \cosh \xi_{6}-k_{7}^{2} s_{7} \cos \xi_{7}\right)}{s_{4} \xi_{4}^{2}+s_{5} \xi_{5}^{2}+s_{6} \cosh \xi_{6}+s_{7} \cos \xi_{7}+m_{2}} \\
& -2 \frac{\left(2 k_{4} s_{4} \xi_{4}+2 k_{5} s_{5} \xi_{5}+k_{6} s_{6} \sinh \xi_{6}-k_{7} s_{7} \sin \xi_{7}\right)^{2}}{\left(l_{4} \xi_{4}^{2}+s_{5} \xi_{5}^{2}+s_{6} \cosh \xi_{6}+s_{7} \cos \xi_{7}+m_{2}\right)^{2}} \\
v & =\frac{2\left(2 k_{4} s_{4} r_{4}+2 k_{5} s_{5} r_{5}+k_{6} s_{6} r_{6} \cosh \xi_{6}-k_{7} s_{7} r_{7} \cos \xi_{7}\right)}{s_{4} \xi_{4}^{2}+s_{5} \xi_{5}^{2}+s_{6} \cosh \xi_{6}+s_{7} \cos \xi_{7}+m_{2}} \\
& -\frac{2\left(2 k_{4} s_{4} \xi_{4}+2 k_{5} s_{5} \xi_{5}+k_{6} s_{6} \sinh \xi_{6}-k_{7} s_{7} \sin \xi_{7}\right)\left(2 s_{4} r_{4} \xi_{4}+2 s_{5} r_{5} \xi_{5}+s_{6} r_{6} \sinh \xi_{6}-s_{7} r_{7} \sin \xi_{7}\right)}{\left(s_{4} \xi_{4}^{2}+s_{5} \xi_{5}^{2}+s_{6} \cosh \xi_{6}+s_{7} \cos \xi_{7}+m_{2}\right)^{2}} .
\end{aligned}
$$

Figs. 4 show the interaction among a rogue wave, a periodic wave and a pair of the stripe solitons via Solutions (15). In Figs. $4\left(a_{1}\right)$ and $\left(b_{1}\right)$, we observe that there is a periodic wave between a pair of the stripe solitons. As $t$ goes on, the rogue wave arises from the one stripe soliton and interacts with the periodic wave. The rogue wave splits into two waves, then the two waves merge into one wave, and finally the rogue wave fuses with the other stripe soliton, as seen in Figs. $4\left(a_{2}\right)-\left(a_{5}\right)$ and $\left(b_{2}\right)-\left(b_{5}\right)$. 


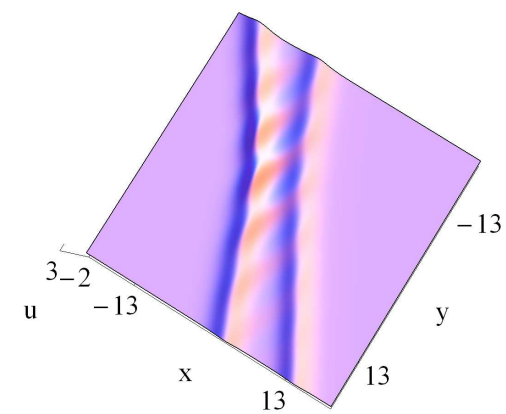

(a) $t=-45$

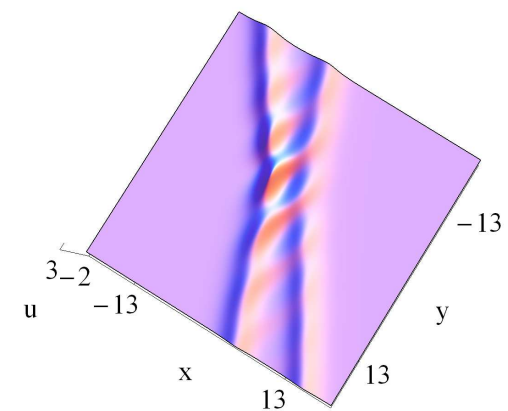

$\left(a_{2}\right) t=-20$

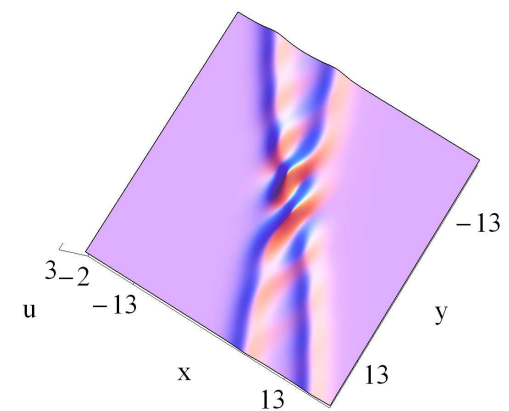

(a) $t=5$

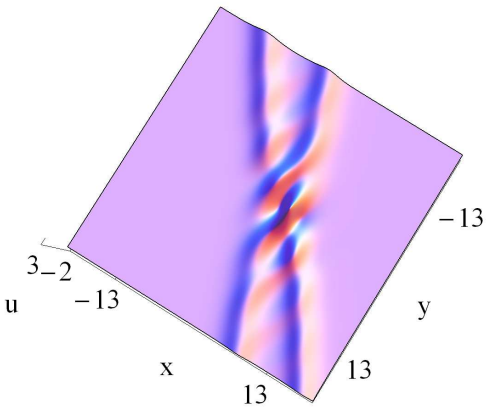

$\left(a_{4}\right) t=30$

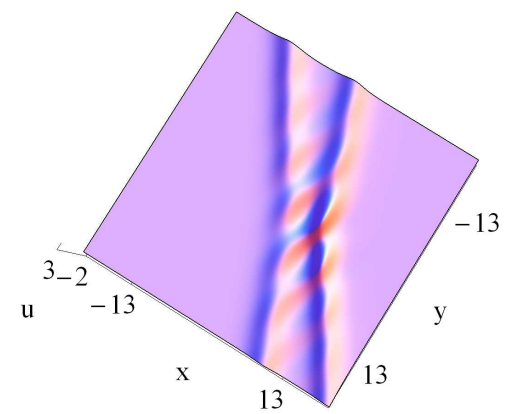

$\left(a_{5}\right) t=55$

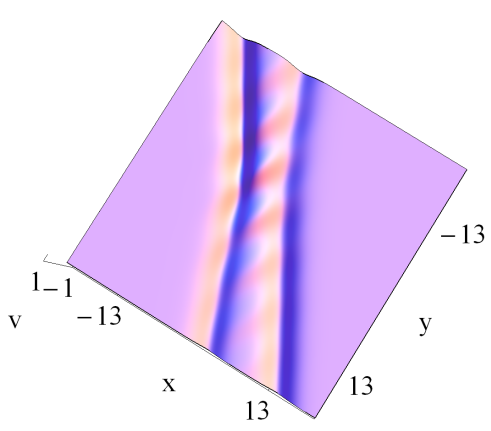

$\left(b_{1}\right) t=-45$

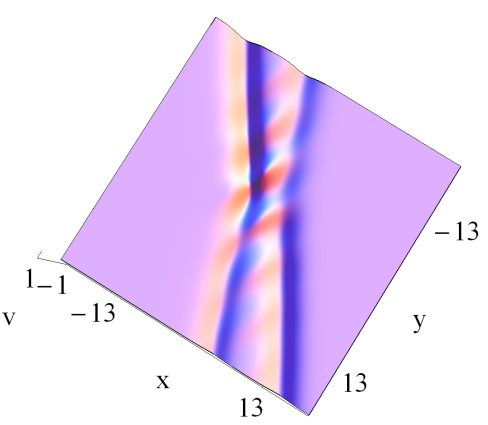

$\left(b_{2}\right) t=-20$

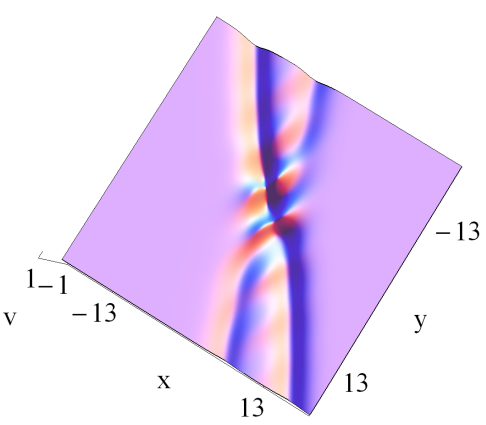

(b) $t=5$

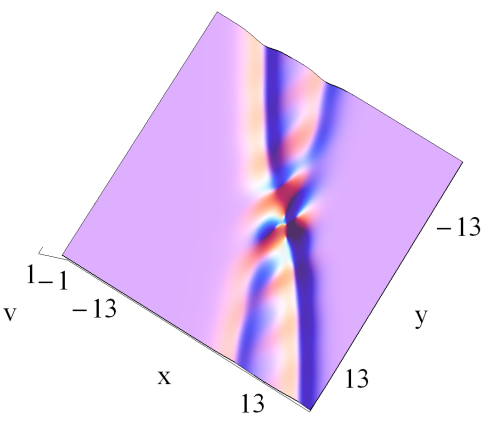

$\left(b_{4}\right) t=30$

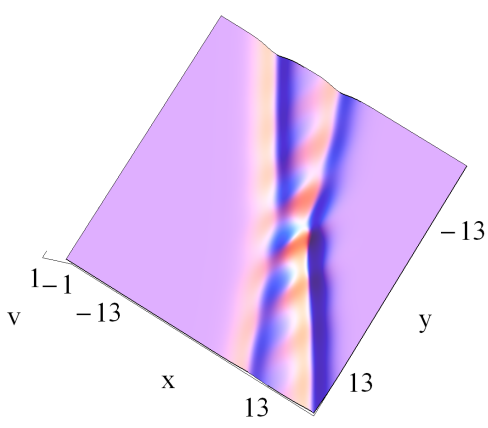

$\left(b_{5}\right) t=55$

Figs. 4. Interaction among a rogue wave, a periodic wave and a pair of the stripe solitons via Solutions (15) with $m_{2}=r_{4}=q_{5}=q_{6}=q_{7}=k_{7}=1, q_{4}=s_{5}=2, s_{7}=3, k_{5}=\frac{1}{3}$ and $s_{6}=\sqrt{ } 10$.

5. Numerical simulations for System (1) 
In the section, for simplicity, we consider the dimensional reduction $\partial_{x}=\partial_{y}$, then System (1) is reduced to

$$
36 w_{t}+w_{x x x x x}+15\left(w w_{x x}\right)_{x}+45 w^{2} w_{x}-5 w_{x x x}-30 w w_{x}-5 w_{x}=0,
$$

with $w$ being the differentiable functions about $x$ and $t$. Next, we will construct the numerical one-soliton solutions for Eq. (16) via a finite difference method [47].

Choosing a finite interval $\Omega=\left[L_{1}, L_{2}\right]$, which is large enough, Eq. (16) with the vanishing boundary condition $\lim _{|x| \rightarrow+\infty}|w|=0$ can be approximated by

$$
\begin{array}{ll}
36 w_{t}+w_{x x x x x}+15\left(w w_{x x}\right)_{x} & +45 w^{2} w_{x}-5 w_{x x x} \\
& -30 w w_{x}-5 w_{x}=0, \quad L_{1}<x<L_{2}, t>0, \\
w(x, t=0)=w_{0}(x), & x \in \Omega, \\
w\left(L_{1}, t\right)=w\left(L_{2}, t\right)=0, & t \geq 0 .
\end{array}
$$

According to Ref. [47], let $h=\frac{L_{2}-L_{1}}{J}$ and $\tau$ be the $x$-direction step size and $t$-direction step size, respectively, then we get the mesh points $x_{j}=L_{1}+j h(j=0,1, \cdots, J)$ and $t_{n}=n \tau$, with $J$ as a positive integer and $n$ as a non-negative positive integer. For simplicity, we introduce some notations as follows [47]:

$$
\begin{aligned}
w_{j}^{n+\frac{1}{2}} & =\frac{w_{j}^{n+1}+w_{j}^{n}}{2}, \quad \delta_{t} w_{j}^{n+\frac{1}{2}}=\frac{w_{j}^{n+1}-w_{j}^{n}}{\tau}, \quad \delta_{x} w_{j}^{n}=\frac{w_{j+1}^{n}-w_{j-1}^{n}}{2 h}, \\
\delta_{x}^{2} w_{j}^{n} & =\frac{w_{j-1}^{n}+w_{j+1}^{n}-2 w_{j}^{n}}{h^{2}}, \quad \delta_{x}^{3} w_{j}^{n}=\frac{-w_{j-2}^{n}+2 w_{j-1}^{n}-2 w_{j+1}^{n}+w_{j+2}^{n}}{2 h^{3}}, \\
\delta_{x}^{5} w_{j}^{n} & =\frac{-w_{j-3}^{n}+4 w_{j-2}^{n}-5 w_{j-1}^{n}+5 w_{j+1}^{n}-4 w_{j+2}^{n}+w_{j+3}^{n}}{2 h^{5}}
\end{aligned}
$$

where $w_{j}^{n}$ denotes the approximate value of $w\left(x_{j}, t_{n}\right)$. Eq. (16) at the point $\left(x_{j}, t_{n+\frac{1}{2}}\right)$ can be written as

$$
\begin{aligned}
& 36 \frac{\partial w}{\partial t}\left(x_{j}, t_{n+\frac{1}{2}}\right)+\frac{\partial^{5} w}{\partial x^{5}}\left(x_{j}, t_{n+\frac{1}{2}}\right)+15 \frac{\partial w}{\partial x}\left(x_{j}, t_{n+\frac{1}{2}}\right) \frac{\partial^{2} w}{\partial x^{2}}\left(x_{j}, t_{n+\frac{1}{2}}\right) \\
& +15 w\left(x_{j}, t_{n+\frac{1}{2}}\right) \frac{\partial^{3} w}{\partial x^{3}}\left(x_{j}, t_{n+\frac{1}{2}}\right)+45 w^{2}\left(x_{j}, t_{n+\frac{1}{2}}\right) \frac{\partial w}{\partial x}\left(x_{j}, t_{n+\frac{1}{2}}\right) \\
& -5 \frac{\partial^{3} w}{\partial x^{3}}\left(x_{j}, t_{n+\frac{1}{2}}\right)-30 w\left(x_{j}, t_{n+\frac{1}{2}}\right) \frac{\partial w}{\partial x}\left(x_{j}, t_{n+\frac{1}{2}}\right)-5 \frac{\partial w}{\partial x}\left(x_{j}, t_{n+\frac{1}{2}}\right)=0 .
\end{aligned}
$$

Based on the Taylor expansion, we have

$$
\begin{array}{lll}
\frac{\partial w}{\partial t}\left(x_{j}, t_{n+\frac{1}{2}}\right)=\delta_{t} w_{j}^{n+\frac{1}{2}}+O\left(\tau^{2}\right), & & \frac{\partial w}{\partial x}\left(x_{j}, t_{n+\frac{1}{2}}\right)=\delta_{x} w_{j}^{n+\frac{1}{2}}+O\left(h^{2}\right), \\
\frac{\partial^{2} w}{\partial x^{2}}\left(x_{j}, t_{n+\frac{1}{2}}\right)=\delta_{x}^{2} w_{j}^{n+\frac{1}{2}}+O\left(h^{2}\right), & & \frac{\partial^{3} w}{\partial x^{3}}\left(x_{j}, t_{n+\frac{1}{2}}\right)=\delta_{x}^{3} w_{j}^{n+\frac{1}{2}}+O\left(h^{2}\right), \\
\frac{\partial^{5} w}{\partial x^{5}}\left(x_{j}, t_{n+\frac{1}{2}}\right)=\delta_{x}^{5} w_{j}^{n+\frac{1}{2}}+O\left(h^{2}\right) . &
\end{array}
$$


Substituting Expressions (20) into Eq. (19), we derive that

$$
\begin{aligned}
& 36 \delta_{t} w_{j}^{n+\frac{1}{2}}+\delta_{x}^{5} w_{j}^{n+\frac{1}{2}}+\frac{15}{2} \delta_{x} w_{j}^{n+\frac{1}{2}}\left(\delta_{x}^{2} w_{j}^{n+1}+\delta_{x}^{2} w_{j}^{n}\right)+\frac{15}{2} w_{j}^{n+\frac{1}{2}}\left(\delta_{x}^{3} w_{j}^{n+1}+\delta_{x}^{3} w_{j}^{n}\right) \\
& +\frac{45}{2}\left(w_{j}^{n+\frac{1}{2}}\right)^{2}\left(\delta_{x} w_{j}^{n+1}+\delta_{x} w_{j}^{n}\right)-5 \delta_{x}^{3} w_{j}^{n+\frac{1}{2}}-15 w_{j}^{n+\frac{1}{2}}\left(\delta_{x} w_{j}^{n+1}+\delta_{x} w_{j}^{n}\right)-5 \delta_{x} w_{j}^{n+\frac{1}{2}}=R_{j}^{n+\frac{1}{2}}
\end{aligned}
$$

where $R_{j}^{n+\frac{1}{2}}=O\left(h^{2}+\tau^{2}\right)$. Omitting $R_{j}^{n+\frac{1}{2}}$, we obtain that

$$
\begin{aligned}
& 36 \delta_{t} w_{j}^{n+\frac{1}{2}}+\delta_{x}^{5} w_{j}^{n+\frac{1}{2}}+\frac{15}{2} \delta_{x} w_{j}^{n+\frac{1}{2}}\left(\delta_{x}^{2} w_{j}^{n+1}+\delta_{x}^{2} w_{j}^{n}\right)+\frac{15}{2} w_{j}^{n+\frac{1}{2}}\left(\delta_{x}^{3} w_{j}^{n+1}+\delta_{x}^{3} w_{j}^{n}\right) \\
& +\frac{45}{2}\left(w_{j}^{n+\frac{1}{2}}\right)^{2}\left(\delta_{x} w_{j}^{n+1}+\delta_{x} w_{j}^{n}\right)-5 \delta_{x}^{3} w_{j}^{n+\frac{1}{2}}-15 w_{j}^{n+\frac{1}{2}}\left(\delta_{x} w_{j}^{n+1}+\delta_{x} w_{j}^{n}\right)-5 \delta_{x} w_{j}^{n+\frac{1}{2}}=0 .
\end{aligned}
$$

Eq. (22) is collapsed to obtain that

$$
\begin{aligned}
& w_{j}^{n+1}+\frac{\tau A^{n+1}}{144 h^{5}}+\frac{5 \tau G B^{n+1}}{96 h^{3}}+\frac{\tau(15 H-10) C^{n+1}}{288 h^{3}}+\frac{\tau\left(45 H^{2}-60 H-20\right) D^{n+1}}{576 h} \\
= & w_{j}^{n}-\frac{\tau A^{n}}{144 h^{5}}-\frac{5 \tau G B^{n}}{96 h^{3}}-\frac{\tau(15 H-10) C^{n}}{288 h^{3}}-\frac{\tau\left(45 H^{2}-60 H-20\right) D^{n}}{576 h} .
\end{aligned}
$$

By the iterative method [47], the iterative scheme for Finite Difference Scheme (23) can be written as

$$
\begin{aligned}
& w_{j}^{n+1, l+1}+\frac{\tau A^{n+1, l+1}}{144 h^{5}}+\frac{5 \tau \tilde{G} B^{n+1, l+1}}{96 h^{3}}+\frac{\tau(15 \tilde{H}-10) C^{n+1, l+1}}{288 h^{3}}+\frac{\tau\left(45 \tilde{H}^{2}-60 \tilde{H}-20\right) D^{n+1, l+1}}{576 h} \\
= & w_{j}^{n}-\frac{\tau A^{n}}{144 h^{5}}-\frac{5 \tau \tilde{G} B^{n}}{96 h^{3}}-\frac{\tau(15 \tilde{H}-10) C^{n}}{288 h^{3}}-\frac{\tau\left(45 \tilde{H}^{2}-60 \tilde{H}-20\right) D^{n}}{576 h},
\end{aligned}
$$

where

$$
\begin{aligned}
& A^{\gamma}=-w_{j-3}^{\gamma}+4 w_{j-2}^{\gamma}-5 w_{j-1}^{\gamma}+5 w_{j+1}^{\gamma}-4 w_{j+2}^{\gamma}+w_{j+3}^{\gamma}, \quad B^{\gamma}=w_{j-1}^{\gamma}+w_{j+1}^{\gamma}-2 w_{j}^{\gamma}, \\
& C^{\gamma}=-w_{j-2}^{\gamma}+2 w_{j-1}^{\gamma}-2 w_{j+1}^{\gamma}+w_{j+2}^{\gamma}, D^{\gamma}=w_{j+1}^{\gamma}-w_{j-1}^{\gamma}, H=w_{j}^{n+1}+w_{j}^{n}, \quad \tilde{H}=w_{j}^{n+1, l+1}+w_{j}^{n}, \\
& G=-w_{j-1}^{n+1}-w_{j-1}^{n}+w_{j+1}^{n+1}+w_{j+1}^{n}, \tilde{G}=-w_{j-1}^{n+1, l+1}-w_{j-1}^{n}+w_{j+1}^{n+1, l+1}+w_{j+1}^{n}, \\
& \gamma=n \text { or } n+1 \text { or } n+1, l+1, w_{j}^{n+1, l=0}=w_{j}^{n}, \quad \lim _{l \rightarrow+\infty} w_{j}^{n+1, l+1}=w_{j}^{n+1},
\end{aligned}
$$

and $l=0,1,2, \cdots$ denotes the iteration time.

From $t_{n}$ to $t_{n+1}$, the iteration stops when $\left|w_{j}^{n+1, l+1}-w_{j}^{n+1, l}\right|<\varepsilon$, with $\varepsilon$ being a given error bond.

We let the error between the analytic solutions $w\left(x_{j}, t_{n}\right)$ and the numerical solutions $w_{j}^{n}$ as

$$
\|E(h, \tau)\|_{\infty}=\max _{\substack{0 \leq j \leq J \\ n \geq 0}}\left|w\left(x_{j}, t_{n}\right)-w_{j}^{n}\right|,
$$


and define

$$
\text { Rate }=\log _{2}\left[\frac{\|E(h, \tau)\|_{\infty}}{\|E(h / 2, \tau / 2)\|_{\infty}}\right]
$$

as an approximation of the rate of convergence when both $h$ and $\tau$ are sufficiently small. As such, if the scheme is the second-order accurate in $x$ direction and the second-order accurate in $t$ direction, the obtained Rate should be 2 .

We choose the analytic one-soliton solution for Eq. (16),

$$
w=\frac{1}{2} \operatorname{sech}^{2}\left[\frac{1}{2}\left(x+\frac{1}{4} t\right)\right] .
$$

Then, we simulate the one-soliton evolution by choosing Solution (27) at $t=0$ as the initial data. In our simulations, we take the interval $\Omega=[-30,30], h=0.2, \tau=0.05$ and error bond $\varepsilon=10^{-8}$ for the iterative computation of Scheme (24).

Fig. 5(a) shows the propagation of the one soliton via Solution (27). Fig. 5(b) displays the numerical one-soliton solution. Fig. $5(c)$ depicts a comparison of the analytic and numerical one-soliton solutions with $t$.

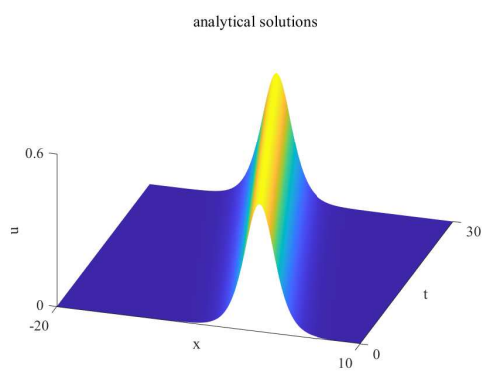

(a)

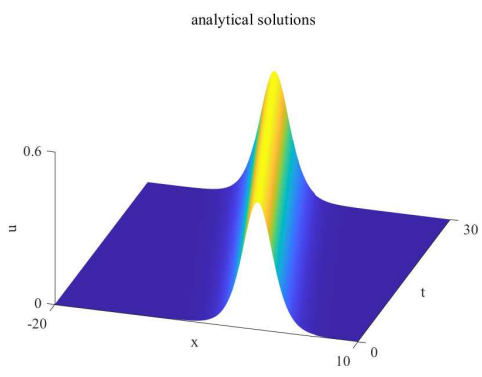

(b)

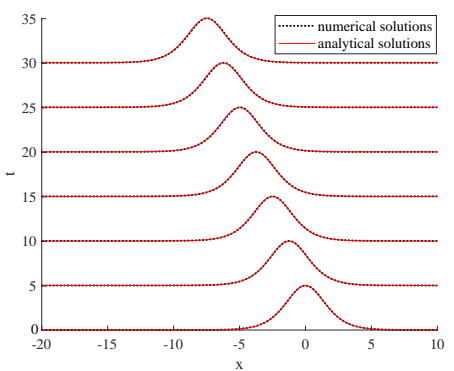

(c)

Figs. 5. (a) Analytical solution of the one soliton via Solution (27); (b) Numerical solution of the one soliton via Eq. (24); (c) Comparison of the analytical one-soliton solution and numerical one-soliton solution .

Table 1: Errors and convergence rates under different steps at $\mathrm{t}=1$

\begin{tabular}{lcc}
\hline$(h, \tau)$ & $\|E(h, \tau)\|_{\infty}$ & Rate \\
\hline$\left(\frac{1}{2}, \frac{1}{500}\right)$ & $3.75727 \times 10^{-3}$ & - \\
$\left(\frac{1}{4}, \frac{1}{1000}\right)$ & $9.79221 \times 10^{-4}$ & 1.93998 \\
$\left(\frac{1}{8}, \frac{1}{2000}\right)$ & $2.49984 \times 10^{-4}$ & 1.96980 \\
$\left(\frac{1}{16}, \frac{1}{4000}\right)$ & $6.21383 \times 10^{-5}$ & 2.00828 \\
\hline
\end{tabular}

Table 1 shows the errors and the convergence rates of the analytic and numerical one-soliton solutions under different $h$ and $\tau$ at $t=1$. From Table 1 , we find that the errors between the 
numerical and analytic one-soliton solutions are small and the convergence rates are close to 2 , which is the result as expected.

\section{Conclusions}

In this paper, symbolic computation has been conducted on a $(2+1)$-dimensional CaudreyDodd-Gibbon-Kotera-Sawada system in fluid mechanics, i.e., System (1). Via the Hirota method, Singular N-Soliton Solutions (5), Shock-Wave Solutions (9), Breather-Stripe Soliton Solutions (12) and Hybrid Solutions (15) have been derived. Via Finite Difference Scheme (23), we have simulated the one-soliton propagation for Eq. (16).

Figs. 1 and 2 have shown the propagations of the singular one soliton and the shock wave, respectively. We have observed that the singular one-soliton solutions are explosive and unstable, but the shock-wave solutions are nonsingular and stable.

Figs. 3 have displayed the propagation of the breather-stripe soliton. We have observed that the breather-stripe soliton moves along the negative direction of the $y$ axis. We have found that the amplitude and shape of the breather-stripe soliton remain invariant during the propagation, as shown in Figs. 3.

Based on Solutions (15), we have investigated the interaction among a rogue wave, a periodic wave and a pair of the stripe solitons, as shown in Figs. 4 . It has been seen that there is a periodic wave between a pair of the stripe solitons, as shown in Fig. $4\left(a_{1}\right)$ and $\left(b_{1}\right)$. As $t$ goes on, we have observed that the rogue wave arises from the one stripe soliton and interacts with the periodic wave, next the rogue wave splits into two waves, then the two waves merge into one wave, and finally the rogue wave fuses with the other stripe soliton, as seen in Figs. 4( $\left.a_{2}\right)-\left(a_{5}\right)$ and $\left(b_{2}\right)-\left(b_{5}\right)$.

Figs. 5(a) and (b) have displayed the analytic one-soliton and numerical one-soliton solutions, respectively. Fig. 5(c) has depicted a comparison of the analytic and numerical solutions with $t$, which indicates that the numerical one-soliton solution which agree with the analytic one-soliton solution. Table 1 has shown the errors and the convergence rates of the analytic and numerical one-soliton solutions under different $h$ and $\tau$ at $t=1$. From Table 1, we have found that the errors between the numerical and analytic one-soliton solutions are small and the convergence rates are close to 2, which is the result as expected.

Hirota method has been used to find the analytic solutions for the NLEEs. Advantage of the Hirota method over the others is that it is more convenient to produce results for certain NLEEs. We expect that the results of this paper will be helpful to the propagation of waves in fluid mechanics.

\section{Acknowledgments}


This work has been supported by the National Natural Science Foundation of China under Grant Nos. 11772017, 11272023 and 11471050, by the Fund of State Key Laboratory of Information Photonics and Optical Communications (Beijing University of Posts and Telecommunications), China (IPOC: 2017ZZ05) and by the Fundamental Research Funds for the Central Universities of China under Grant No. 2011BUPTYB02.

Data Availability Some or all data, models or code generated or used during the study are available from the corresponding author by request.

Compliance with ethical standards Research does not involve Human Participants and/or Animals.

Conflict of interest The authors declare that they have no conflict of interest.

\section{References}

[1] Karabut, E.A., Zhuravleva, E.N., Zubarev, N.M.: Application of transport equations for constructing exact solutions for the problem of motion of a fluid with a free boundary. J. Fluid Mech. 890, A13 (2020)

[2] Morris, J.F.: Toward a fluid mechanics of suspensions. Phys. Rev. Fluids 5, 110519 (2020)

[3] Aref, H., Balachandar, S.: A First Course in Computational Fluid Dynamics. Cambridge Univ. Press, Cambridge (2018)

[4] Falkovich, G.: Fluid Mechanics. Cambridge Univ. Press, Cambridge (2018)

[5] Wazwaz, A.M., Kaur, L.: New integrable Boussinesq equations of distinct dimensions with diverse variety of soliton solutions. Nonlinear Dyn. 97, 83-94 (2019)

[6] Mabrouk, S.M., Rashed, A.S.: $N$-Solitons, kink and periodic wave solutions for $(3+1)$ dimensional Hirota bilinear equation using three distinct techniques. Chin. J. Phys. 60, 48-60 (2019)

[7] Liu, W.H., Zhang, Y.F.: High-order rational solutions and rogue wave for the (2+1)dimensional nonlinear Schrödinger equation. Phys. Scripta 95, 045204 (2020)

[8] Yusuf, A., Sulaiman, T.A., Bayram, M.: Breather wave, lump-periodic solutions and some other interaction phenomena to the Caudrey-Dodd-Gibbon equation. Eur. Phys. J. Plus 135, 1-8 (2020)

[9] Kaur, L., Wazwaz, A.M.: Lump, breather and solitary wave solutions to new reduced form of the generalized BKP equation. Int. J. Numer. Method H. 29, 569-579 (2019) 
[13] Feng, Y.J., Gao, Y.T., Li, L.Q., Jia T.T.: Bilinear form, solitons, breathers and lumps of a $(3+1)$-dimensional generalized Konopelchenko-Dubrovsky-Kaup-Kupershmidt equation in ocean dynamics, fluid mechanics and plasma physics. Eur. Phys. J. Plus 135, $272(2020)$

[14] Feng, Y.J., Gao, Y.T., Jia T.T., Li, L.Q.: Soliton interactions of a variable-coefficient three-component AB system for the geophysical flows. Mod. Phys. Lett. B 33, 1950354 (2019)

[15] Su, J.J., Gao, Y.T., Deng, G.F., Jia, T.T.: Solitary waves, breathers, and rogue waves modulated by long waves for a model of a baroclinic shear flow. Phys. Rev. E 100, 042210 (2019)

[16] Su, J.J., Gao, Y.T., Ding, C.C.: Darboux transformations and rogue wave solutions of a generalized AB system for the geophysical flows. Appl. Math. Lett. 88, 201-208 (2019)

[17] Hu, L., Gao, Y.T., Jia, S.L., Su, J.J., Deng, G.F.: Solitons for the (2+1)-dimensional Boiti-Leon-Manna-Pempinelli equation for an irrotational incompressible fluid via the Pfaffian technique. Mod. Phys. Lett. B 33, 1950376 (2019)

[18] Hu, L., Gao, Y.T., Jia, T.T., Deng, G.F., Li, L.Q.: Higher-order hybrid waves for the (2+1)-dimensional Boiti-Leon-Manna-Pempinelli equation for an irrotational incompressible fluid via the modified Pfaffian technique. Z. Angew. Math. Phys. 72, 75 (2021)

[19] Jia, T.T., Gao, Y.T., Yu, X., Li, L.Q.: Lax pairs, Darboux transformation, bilinear forms and solitonic interactions for a combined Calogero-Bogoyavlenskii-Schiff-type equation. Appl. Math. Lett. 114, 106702 (2021)

[20] Jia, T.T., Gao, Y.T., Deng, G.F., Hu, L.: Quintic time-dependent-coefficient derivative nonlinear Schrödinger equation in hydrodynamics or fiber optics: bilinear forms and dark/anti-dark/gray solitons. Nonlinear Dyn. 98, 269-282 (2019) 
[21] Deng, G.F., Gao, Y.T., Ding, C.C., Su, J.J.: Solitons and breather waves for the generalized Konopelchenko-Dubrovsky-Kaup-Kupershmidt system in fluid mechanics, ocean dynamics and plasma physics. Chaos Solitons Fract. 140, 110085 (2020)

[22] Deng, G.F., Gao, Y.T., Su, J.J., Ding, C.C., Jia, T.T.: Solitons and periodic waves for the $(2+1)$-dimensional generalized Caudrey-Dodd-Gibbon-Kotera-Sawada equation in fluid mechanics. Nonlinear Dyn. 99, 1039-1052 (2020)

[23] Li, L.Q., Gao, Y.T., Hu, L., Jia, T.T., Ding, C.C., Feng, Y.J.: Bilinear form, soliton, breather, lump and hybrid solutions for a $(2+1)$-dimensional Sawada-Kotera equation. Nonlinear Dyn. 100, 2729-2738 (2020)

[24] Ding, C.C., Gao, Y.T., Deng, G.F., Wang, D.: Lax pair, conservation laws, Darboux transformation, breathers and rogue waves for the coupled nonautonomous nonlinear Schrödinger system in an inhomogeneous plasma. Chaos Solitons Fract. 133, 109580 (2020)

[25] Ding, C. C., Gao, Y. T., Hu, L., Deng, G. F., Zhang, C. Y.: Vector bright soliton interactions of the two-component AB system in a baroclinic fluid, Chaos Solitons Fract. 142, $110363(2021)$

[26] Liu, F. Y., Gao, Y. T., Yu, X., Hu, L., Wu, X. H.: Hybrid solutions for the (2+1)dimensional variable-coefficient Caudrey-Dodd-Gibbon-Kotera-Sawada equation in fluid mechanics, Chaos Solitons Fract. 152, 111355 (2021)

[27] Liu, F. Y., Gao, Y.T., Yu, X., Li, L. Q., Ding, C. C., Wang, D.: Lie group analysis and analytic solutions for a $(2+1)$-dimensional generalized Bogoyavlensky-Konopelchenko equation in fluid mechanics and plasma physics. Eur. Phys. J. Plus 136, 656 (2021)

[28] Gao, X. Y., Guo, Y. J., Shan, W. R.: Optical waves/modes in a multicomponent inhomogeneous optical fiber via a three-coupled variable-coefficient nonlinear Schrödinger system, Appl. Math. Lett. 120, 107161 (2021)

[29] Li, L. Q., Gao, Y. T., Yu, X., Jia, T. T., Hu, L., Zhang, C. Y.: Bilinear forms, bilinear Bäcklund transformation, soliton and breather interactions of a damped variable-coefficient fifth-order modified Korteweg-de Vries equation for the surface waves in a strait or large channel, Chin. J. Phys. (2022) in press, https://doi.org/10.1016/j.cjph.2021.09.004

[30] Wang, X., Chen, Y.: Darboux transformations and $N$-soliton solutions of two (2+1)dimensional nonlinear equations. Commun. Theor. Phys. 61, 423 (2014) 
[31] Yang, Z.H.: A series of exact solutions of $(2+1)$-dimensional CDGKS equation. Commun. Theor. Phys. 46, 807 (2006)

[32] Zhuang, J.H., Liu, Y.Q., Chen, X., Wu, J.J., Wen, X.Y.: Diverse solitons and interaction solutions for the (2+1)-dimensional CDGKS equation. Mod. Phys. Lett. B 33, 1950174 (2019)

[33] Cao, C.W., Wu, Y.T., Geng, X.G.: On quasi-periodic solutions of the $2+1$ dimensional Caudrey-Dodd-Gibbon-Kotera-Sawada equation. Phys. Lett. A 256, 59-65 (1999)

[34] Lü, N., Mei, J.Q., Zhang, H.Q.: Symmetry reductions and group-invariant solutions of $(2+1)$-dimensional Caudrey-Dodd-Gibbon-Kotera-Sawada equation. Commun. Theor. Phys. 53, 591 (2010)

[35] Fang, T., Gao, C.N., Wang, H., Wang, Y.H.: Lump-type solution, rogue wave, fusion and fission phenomena for the (2+1)-dimensional Caudrey-Dodd-Gibbon-KoteraSawada equation. Mod. Phys. Lett. B 33, 1950198 (2019)

[36] Li, W.T., Zhang, Z., Yang, X.Y., Li, B.: High-order breathers, lumps and hybrid solutions to the $(2+1)$-dimensional fifth-order KdV equation. Int. J. Mod. Phys. B 33, 1950255 (2019)

[37] Meng, X.H.: The periodic solitary wave solutions for the $(2+1)$-dimensional fifth-order KdV equation. J. Appl. Math. Phys. 2, 639-643 (2014)

[38] Kang, X.R., Xian, D.Q., Dai, Z.D.: Non-traveling wave solutions for the (2+1)-D Caudrey-Dodd-Gibbon-Kotera-Sawada equation. Int. J. Numer. Method. H. 25, 617$628(2015)$

[39] Zhuang, J.H., Liu, Y.Q., Wu, J.J., Zhuang, P., Chen, X., Wen, X.Y.: The High Order Interaction Solutions Comprising Lump Solitons for the $(2+1)$-dimensional Caudrey-Dodd-Gibbon-Kotera-Sawada Equation. Authorea Preprints, in press, (2020). doi: 10.22541/au.160137914.44290163.

[40] Tang, Y.N., Tao, S.Q., Guan, Q.: Lump solitons and the interaction phenomena of them for two classes of nonlinear evolution equations. Comput. Math. Appl. 72, 2334-2342 (2016)

[41] Wang, T.T., Liu, X.Q., Yu, J.Q.: Symmetries, exact solutions and conservation laws of Caudrey-Dodd-Gibbon-Kotera-Sawada equation. Chinese J. Quantum Elect. 28, 385 (2011) 
[42] Zhuang, J.H., Liu, Y.Q., Zhuang, P.: Variety interaction solutions comprising lump solitons for the (2+1)-dimensional Caudrey-Dodd-Gibbon-Kotera-Sawada equation. AIMS Math. 6, 5370-5386 (2021)

[43] Hirota, R.: The direct method in soliton therory. Cambridge University Press, Cambridge (2004)

[44] Lu, D., Tariq, K.U., Osman, M.S., Baleanu, D., Younis, M., Khater, M.M.: New analytical wave structures for the $(3+1)$-dimensional Kadomtsev-Petviashvili and the generalized Boussinesq models and their applications. Results Phys. 14, 102491 (2019)

[45] Gai, L.T., Ma, W.X., Li, M.C.: Lump-type solution and breather lump-kink interaction phenomena to a $(3+1)$-dimensional GBK equation based on trilinear form. Nonlinear Dyn. 100, 2715-2727 (2020)

[46] Ullah, M.S., Roshid, H.O., Ma, W.X., Ali, M.Z., Rahman, Z.: Interaction phenomena among lump, periodic and kink wave solutions to a $(3+1)$-dimensional Sharma-TassoOlver-like equation. Chin. J. Phys. 68, 699-711 (2020)

[47] Li, S.C., Li, X.G., Cao, J.J., Li, W.B.: High-order numerical method for the derivative nonlinear Schrödinger equation. Int. J. Model. Simul. Sci. Comput. 8, 1750017 (2017) 